\title{
Regulating the Expression of Malignancy
}

MALIGNANCY is a heritable state; cells transformed by chemicals or by tumour viruses, for example, retain their malignancy during prolonged passage in culture and, in general, there is a satisfying correlation between the ability of cultivated transformed cells to induce tumours when inoculated into susceptible animals and their lack of growth control exhibited under a variety of tissue culture regimens. Both a cell's loss of growth control in vitro and its malignancy in vivo can, however, be reversed; by applying selection pressures to cultures of transformed malignant cells it is possible to select socalled revertants - cells which behave in culture as if they were normal, even though in the case of viral transformation the revertants retain the tumour virus genome. Such revertants, which in tissue culture exhibit growth control comparable to that of normal cells, are usually found to be no more malignant than untransformed cells when they are inoculated into susceptible animals. It is obvious therefore that the expression of transformation and malignancy can be modulated or suppressed. A cell which has a tumour virus genome(s) stably associated with and probably integrated in one of its chromosomes, or some other oncogene, may yet have the phenotype of a normal, untransformed cell.

Several research groups have reported in Nature and elsewhere experiments, all of which indicate that malignancy can be suppressed by changing the chromosomal constitution of a cell. Harris and his colleagues, for example (Nature, 223, $368 ; 1969$ ), suppressed the malignancy of a line of mouse cells by fusing them to nonmalignant mouse cells; Pollack and his collaborators (Nature, 228, $938 ; 1970$ ) observed that when mouse 3T3 cells transformed by SV40 revert to a normal phenotype their chromosome number increases from a median value of 75 to 118. Likewise Rabinowitz and Sachs (Nature, $225,136 ; 1970)$ correlated the reversion of hamster embryo cells transformed by polyoma virus with an increase in the number of chromosomes. From this they postulated that the balance between two sorts of chromosomes - those carrying factors for the expression and those with factors for the suppression of transforming genesdetermines whether or not the malignant phenotype is expressed. On page 511 of this issue of Nature, Hitotsumachi, Rabinowitz and Sachs describe new experiments designed to validate this suggestion.

They have now examined the chromosome complements of twenty-eight revertants of hamster embryo cells transformed by polyoma virus and find that reversion can be associated with a decrease as well as an increase in the chromosome number. Furthermore, reversion is not an all or nothing phenomenon, for the growth properties in vitro and the ability to induce tumours of revertants may fall anywhere in the spectrum between normal and malignant cells. That is not, of course, surprising if it is the balance between two sets of chromosomes carrying opposing factors that controls the expression of transforming genes. But if this hypothesis is correct there should be differences between the sorts of chromosomes as well as the number of chromosomes in normal, transformed and revertant cells.

Using the size, shape and position of the centromere as indices, Hitotsumachi et al. divided the chromosomes of their revertants into a series of groups and compared these groups with their counterparts in normal and malignant cells. They believe that they have found that reversion, a decrease in the expression of the transformed cell phenotype, is correlated with a decrease in the number of chromosomes in one particular group, group 5. Chromosomes of this group therefore probably carry factors stimulating the expression of transforming genes. By contrast, chromosomes of group 9, which are present in revertant cells but not normal or transformed cells, probably carry factors which suppress the expression of malignancy. Because the appearance of group 9 chromosomes is associated with a decrease in the number of chromosomes in groups 6 and 7, Sachs and his colleagues suggest that the group 9 chromosomes of revertants are formed from groups 6 and 7 chromosomes, the latter bearing suppressor factors in normal and malignant cells.

Needless to say, experiments of the sort reported by Sachs and his colleagues depend absolutely on the precision with which individual chromosomes can be distinguished. And no doubt they would be among the first to admit that the currently available techniques for staining chromosomes to reveal their individuality leave much to be desired; a glance at the figures which illustrate their report and those of Rowley and Bodmer and Yunis and his colleagues (see pages 503 and 532) in this issue of Nature is enough to convince anybody of the inherent tedium of such experiments. New techniques are, however, coming along which should increase the certainty with which any particular chromosome can be picked out.

Rowley and Bodmer, for example, report the correlated use of three different staining techniques to identify the characteristic distribution of regions of heterochromatic. satellite DNA along the length of individual mouse chromosomes. Staining with aceto-orcein reveals the centromere and other characteristic constrictions along the arms of chromosomes. Fluorescence microscopy of mouse chromosomes stained with quinacrine mustard, a technique Caspersson and his colleagues have developed, reveals the centromere and these same regions of the chromosome as bands of reduced fluorescence. This technique depends on the binding of quinacrine mustard, which can be made to fluoresce, to guanine residues and so it reveals reproducibly regions of the chromosome rich or poor in this base.

Finally, Rowley and Bodmer have denatured and renatured chromosomal DNA in situ using the same preparations that they previously stained with quinacrine mustard. They then stained the cells with Giemsa; the centromere and the same heterochromatic regions which had been revealed by the two other techniques were again revealed, this time more intensely stained than the rest of the chromosome.

These three methods reveal the pattern of distribution of heterochromatic, satellite DNA, in other words the signature, of individual chromosomes. And as Rowley and Bodmer comment, the fluorescent banding technique, in particular, should prove to be extremely valuable in following the chromosomal evolution of cells in culture and in "identifying those mouse chromosomes which may carry genes for repressing malignancy". That is something which Sachs and his colleagues and others will doubtless be quick to follow up. 